\title{
Multidisciplinary Approach for Liver Transplantation in Patients with Nonalcoholic Steatohepatitis: It Takes a Village
}

\author{
Eugenia Tsai, MD ${ }^{1,2, *}$ Ronit Patnaik, MD ${ }^{2, *}$ Naim Alkhouri, MD ${ }^{1,2}$
}

${ }^{1}$ Texas Liver Institute, University of Texas Health San Antonio, San Antonio, Texas

2 Liver Transplantation Center, University of Texas Health San Antonio, San Antonio, Texas

Dig Dis Interv 2020;4:19-30.
Address for correspondence Naim Alkhouri, MD, Texas Liver Institute, University of Texas Health San Antonio, 607 Camden Street,

San Antonio, TX 78215 (e-mail: alkhouri@txliver.com).

\begin{abstract}
Keywords

- nonalcoholic fatty liver disease

- nonalcoholic steatohepatitis

- metabolic syndrome

- liver transplant

- recurrent NAFLD

- de novo NAFLD

Nonalcoholic fatty liver disease (NAFLD) is the leading cause of liver disease in Western countries, and its aggressive form, nonalcoholic steatohepatitis (NASH), is a leading cause of cirrhosis and end-stage liver disease. The total number of new liver transplantation waitlist registrants with NASH continues to increase rapidly, making NASH the second most common indication for liver transplantation. Compared with recipients for other etiologies, patients with NASH often have higher rates of obesity, diabetes, dyslipidemia, hypertension, kidney disease, and cardiac disease. Many of these medical comorbidities are independently associated with increased preoperative risk and both short- and long-term complications. The presence of these particular risk factors necessitates the need for early detection, medical optimization, and careful preoperative care. Bariatric surgery is an effective strategy for weight loss and ultimately reducing obesity-related medical complications. In select patients, bariatric surgery-before, during, or after liver transplantation-may be useful to help improve posttransplant outcomes. NAFLD/NASH can present after liver transplantation and occurs as either recurrent or de novo disease. Posttransplant NAFLD occurs in the setting of metabolic syndrome, immunosuppression use, and genetic determinants. Future studies and efforts should focus on optimizing medical management strategies to further improve transplant outcomes in patients with NAFLD.
\end{abstract}

Nonalcoholic fatty liver disease (NAFLD) is the leading cause of liver disease in North America. NAFLD is an umbrella term, encompassing a wide histological spectrum of disease severity, for which definitive diagnosis requires histology. ${ }^{1}$ The disease spectrum ranges from simple steatosis, which is defined by the presence of steatosis in at least $5 \%$ of hepatocytes in the absence of other causes (e.g., alcohol or medications), to nonalcoholic steatohepatitis (NASH), which is defined by the presence of hepatic fat accumulation,

\footnotetext{
First co-authors.
}

received

October 11, 2019 accepted after revision January 5, 2020

published online

March 2, 2020 inflammation, and ballooning of hepatocytes, to fibrosis and ultimately cirrhosis. In a small proportion of patients, NASH cirrhosis can lead to complications including endstage liver disease (ESLD) and hepatocellular carcinoma (HCC).

Without any U.S. Food and Drug Administration (FDA) approved pharmacological treatments for NAFLD, the disease spectrum is quickly emerging as a top indication for liver transplant (LT). ${ }^{2}$ In the 2015 United Network for Organ Sharing (UNOS) registry, NASH surpassed hepatitis C virus (HCV) infection as the leading indication for LT in patients

Copyright $\odot 2020$ by Thieme Medical Publishers, Inc., 333 Seventh Avenue, New York, NY 10001, USA.

DOI https://doi.org/ Transplant Surgery; Guest Editor, Koji Hashimoto, MD, PhD 10.1055/s-0040-1705103. ISSN 2472-8721. 
under the age of 50 years. ${ }^{3}$ It is predicted to become the leading cause of LT by $2030{ }^{4}$

Unfortunately, the increase in obesity and insulin resistance that leads to NAFLD also means that the potential donor pool of livers available for transplant will only continue to decrease in the future. In addition, once these patients undergo an LT, due to their medical comorbidities, they are at an increased risk of cardiac events, sepsis, and renal failure. Pre- and posttransplant management needs to be specialized for patients with $\mathrm{NASH}^{5}$

\section{NAFLD/NASH Pathophysiology and Epidemiology}

\section{Pathophysiology}

The exact pathophysiological mechanisms of NAFLD still remain incompletely understood; however, the disease process is affected by insulin resistance, lipids, nutritional factors, physical activity, intestinal microbiota, and genetic determinants. ${ }^{6}$

The two-hit hypothesis postulates that the first hit is the intrahepatic accumulation of fatty acids, which then increases the susceptibility of the hepatocytes to a "second hit," which includes oxidative stress, mitochondrial dysfunction, proinflammatory cytokines, and gut dysbiosis)., ${ }^{7,8}$ The two-hit hypothesis is now considered outdated and has been replaced by the multiple parallel-hit hypothesis to explain the natural history and pathogenesis of NAFLD. The multiple-hit pathogenesis suggests that multiple insults act together in genetically predisposed patients. ${ }^{9,10}$

\section{Epidemiology}

Historically, chronic HCV infection was the most common cause of hepatic decompensation leading to HCC and LT. ${ }^{11}$ However, in recent years, due to the advent of curative direct antiviral agents (DAA), there has been a sharp decrease in the proportion of candidates with HCV. In contrast, the proportion of candidates with NASH has demonstrated a tremendous increase over the last decade. ${ }^{12}$ This increase parallels the rising prevalence of type 2 diabetes mellitus (T2DM) and obesity.

Metabolic syndrome (MetS) is a cluster of metabolic abnormalities that is a precursor to cardiovascular disease (CVD) and is a significant risk factor for NAFLD/NASH. In 2005, the American Heart Association and the National Heart, Lung, and Blood Institute updated the previous 2001 National Cholesterol Education Program (NCEP) Adult Treatment Panel III (ATP III) definition of MetS. According to the NCEP ATP III definition, MetS is present if any three of the following five criteria are present: (1) obesity (waist circumference $>40$ inches in males or $>35$ inches in females; (2) hyperglycemia (fasting glucose $\geq 100 \mathrm{mg} / \mathrm{dL}$ or on medication); (3) dyslipidemia (triglyceride $\geq 150 \mathrm{mg} / \mathrm{dL}$ or on medication); (4) dyslipidemia (second, separate criteria) (high-density lipoprotein cholesterol $<40 \mathrm{mg} / \mathrm{dL}$ in males or $<50 \mathrm{mg} / \mathrm{dL}$ in females or on medication; (5) hypertension ( $>130 \mathrm{~mm} \mathrm{Hg}$ systolic or $>85 \mathrm{~mm} \mathrm{Hg}$ diastolic or on medication). ${ }^{13}$
The prevalence of NAFLD worldwide is estimated at 9 to $50 \%$ in the adult population, whereas the prevalence of NAFLD in the United States is reported at 10 to $35 \%{ }^{14}$ The majority of patients with NAFLD have just simple steatosis; however, analysis of NAFLD cohorts using paired liver biopsies suggest that one-third of patients with NAFL/NASH have progressive fibrosis, whereas one-fifth of patients will have some regression. ${ }^{15}$ One global meta-analysis demonstrated fibrosis progression in NASH to be as high as $41 \%{ }^{16} \mathrm{NASH}$ progression to cirrhosis and HCC has an estimated prevalence of 3 to $5 \%$ in the adult population, whereas cirrhosis is observed in up to $20 \%$ of patients with NASH in a 10 -year period. ${ }^{17}$ It is projected that by 2030 , the incidence of decompensated NASH cirrhosis and HCC in the setting of NAFLD/NASH to increase by 168 and $137 \%$, respectively. ${ }^{18}$ In patients with cirrhosis due to NASH, the 10-year mortality from liver failure occurs in 30 to $40 \%$ of patients. ${ }^{19}$

NAFLD-associated HCC occurs in patients with cirrhosis as a potential complication of the disease. In developed countries, the rising incidence of HCC is thought to directly parallel the increasing prevalence of Mets. ${ }^{20}$ In a study of 4,929 cases of HCC between 2004 and 2009, 14.1\% of HCC were due to NAFLD, with an annual increase of $9 \%{ }^{21}$

Diabetes and obesity are associated risk factors for HCC in patients. $^{22}$ While most cases of HCC are associated with cirrhosis, HCC can occur in the absence of cirrhosis in patients with NAFLD, thus leading to a later diagnosis. NAFLD-HCC is more often diagnosed at a later stage with larger tumors when compared with those with cirrhosis and HCC. $^{23}$

\section{Nonalcoholic Steatohepatitis and Transplant}

Based on national registries, the greatest increase in new LT waitlist registrations from 2004 to 2013 was in candidates with NASH; the increase, by $170 \%$, far exceeded registrants with alcohol-related liver disease (increased by $45 \%$ ) and HCV (increased by $14 \%){ }^{24}$

In $2015,10,630$ new candidates were added to the LT waitlist, and NASH was the second leading cause of liver disease among the candidates. ${ }^{11}$ This rising incidence is seen in various subgroups. NASH has become the leading indication for LT in women; from 2004 to 2016, there has been a $91 \%$ increase in female transplant recipients with $\mathrm{NASH}^{25}$ Even in the baby boomer generation (persons born between 1945 and 1965), which was considered the hepatitis C generation, the proportion of patients with NASH added to LT waitlist has demonstrated incremental growth, up to $83.2 \%$ in 2015 , compared with $60.6 \%$ in $2004(p<0.01) .{ }^{26}$ The proportion of baby boomer LT recipients with NASH also increased incrementally, coming in second after recipients with $\mathrm{HCV}^{27}$

While the number of waitlist registrants with NASH is rapidly increasing, earlier studies demonstrated that patients with NASH are less likely to receive an LT when compared with patients with HCV; the NASH patients were ultimately more likely to be delisted or die before receiving an LT. ${ }^{28}$ However, in an analysis of the UNOS data from 2002 to 2016, Thuluvath et al demonstrated that patients with 
NASH are not disadvantaged by higher waitlist removal or lower transplant rates when compared with their counterparts with other liver disease etiologies. The cumulative incidence of death or deterioration was similar for patients with NASH cirrhosis, cryptogenic cirrhosis, alcohol-related cirrhosis, and autoimmune hepatitis $(29,28,28$, and $24 \%$, respectively) along with similar rates of transplantation (48-54\%) across all groups. ${ }^{29}$

\section{Pretransplant Considerations}

There is growing evidence that NAFLD is a multisystem disease and therefore the clinical burden extends beyond the liver alone. NAFLD is closely related to T2DM, CVD, and chronic kidney disease (CKD), and many patients with NAFLD have the aforementioned comorbidities. Recognition of these medical conditions is important to help medically optimize patients in the pretransplant setting.

\section{Obesity}

The prevalence of obesity in the United States is currently estimated to be approximately $35 \%{ }^{30}$ In patients with obesity, the prevalence of NAFLD is approximately 70 to $80 \%$, whereas the prevalence of NASH is estimated at around 15 to $20 \%{ }^{31}$ The current epidemic of obesity has impacted patients with ESLD and may be either the primary cause of liver disease or at least a contributing factor in the patient's disease. As the rates of obesity continue to rise, the prevalence of NAFLD increases in parallel.

Obesity is strongly associated with diabetes, heart disease, and cancer, which are leading causes of morbidity and mortality post-LT. ${ }^{32}$ Studies on the impact of obesity on posttransplant outcomes have been inconsistent. In an early study, Leonard et al. demonstrated that obese patients require greater operative time and blood product usage and had a higher risk of complications including increased intensive care unit length of stay, wound and intra-abdominal infections, and biliary complications. ${ }^{33}$ Later studies have demonstrated that obese and morbidly obese patients may be transplanted with short-term outcomes similar to nonobese patients. ${ }^{34,35}$ While the short-term outcomes of LT may be similar in those who are obese and nonobese, the long-term impact of obesity on post-LT outcomes including recurrence of NASH is becoming increasingly evident. ${ }^{36}$

Despite the technical surgical challenges and potential medical complications, obesity alone should not be an absolute contraindication to LT. Many programs aim to optimize recipients' body mass index (BMI) to allow for the best outcomes. Bariatric surgery has long been proven to be an effective weight loss strategy that can also reduce obesity-related complications.

\section{Bariatric Surgery and NAFLD/NASH}

The role of bariatric surgery in patients with liver disease continues to evolve. Weight loss induced by bariatric surgery has demonstrated some promise as a treatment option for NAFLD. Studies have demonstrated dramatic improvement in, even resolution of, NASH in obese patients who undergo bariatric surgery. ${ }^{37-39}$ In a recent, large propensity-matched cohort study, results supported older studies stating that bariatric surgery in obese patients significantly reduce the incidence of NASH. ${ }^{40}$ Various bariatric surgeries are available, and in comparative studies on Roux-en-Y gastric bypass (RYGB) and laparoscopic sleeve gastrectomy (LSG), there were no significant differences in improvement in liver histology of NASH between the two surgical methods. ${ }^{41,42}$

In a patient with cirrhosis, particularly decompensated cirrhosis, bariatric surgery is associated with a higher mortality. ${ }^{43}$ However, in posttransplant patients, it may be associated with technical difficulty due to adhesions and issues of long-term immunosuppression use. ${ }^{44}$

\section{Bariatric Surgery and Liver Transplantation}

RYGB provides more effective weight loss than LSG. ${ }^{45}$ In the study by Nickel et al, there was a significant improvement of NAFLD 12.5 months after bariatric surgery, with the median fibrosis stage in transient elastography (TE) decreasing from $\mathrm{F} 3$ to $\mathrm{F} 1{ }^{45}$ The high preoperative fibrosis stages are in line with findings in the study by Luger et al, which showed a prevalence, confirmed by liver biopsy, of NASH in 72\%, simple steatosis in $11 \%$, and a normal liver in $17 \%$ of bariatric surgery patients. $^{46}$

However, since LSG results in fewer complications, it is increasingly the preferred choice in complex patients such as LT recipients. ${ }^{47}$ Concerns with altered drug absorption and the need for higher doses of immunosuppression along with hypovitaminosis associated with RYGB also support the use of LSG. ${ }^{44,48}$ Another benefit of LSG compared with RYGB is preserved access to the biliary tree for a potential future need for endoscopic evaluation of the allograft liver. Hence, most studies have focused on the LSG before or after LT.

The optimal time for bariatric surgery in this population remains undefined. Bariatric surgery has been described in patients before, during, and after LT.

In 2013, Heimbach et al presented data on a small, randomized, single-center study demonstrating that LT combined with LSG for patients with ESLD and obesity $\left(\mathrm{BMI}>35 \mathrm{~kg} / \mathrm{m}^{2}\right)$ was effective for weight loss', with significant decrease in post-LT metabolic complications including posttransplant hepatic steatosis or diabetes when compared with LT with medically managed weight loss. ${ }^{49}$ Over a mean follow-up period of 35 months, there were three deaths and three graft losses in the control group, and no deaths or graft losses in the combined surgery arm, although one patient did develop early graft dysfunction and a subsequent leak from the gastric staple line, necessitating multiple reoperations. Notably, the noninvasive management of obesity, with dedicated obesity programs centered on dietary education, was effective in reducing patient weight and BMI at the time of transplant, indicating that weight loss is achievable without surgery and with medical management through intensive education and counseling.

The largest series on simultaneous LT with LSG is by Zamora-Valdes et al. In their study, out of Mayo Clinic, the group demonstrated a total body weight loss percentage of $36 \%$ in 12 months, but an overall complication rate nearing 
$50 \%$ and two postoperative deaths. ${ }^{50}$ However, morbidity and mortality were likely associated with LT and not LSG. The complications ranged from infection to hepatic artery thrombosis (HAT) and allograft failure.

Obesity confers an increase in the prothrombotic state and is known as a predisposing factor for thromboembolic events. In obese individuals, there is an up to fivefold increased risk of deep venous thrombosis and pulmonary embolisms with increasing BMI. ${ }^{51}$ HAT is the most feared and common vascular complication in LTs, affecting 2 to $12 \%$ of transplants, and the leading cause of graft failure and mortality. ${ }^{52}$ Risk factors for HAT are related to surgical technique, ABO blood group incompatibility, prolonged cold ischemia time of the allograft, and rejection. Pediatric LTs are also more susceptible to HAT, likely due to small vessel caliber. ${ }^{53}$ In the study by Zamora-Valdes et al, 2 of 29 patients developed postoperative HAT, which corresponds with the published percent of LT patients that develop HAT. ${ }^{50}$ Hence, it is difficult to say if the increased hypercoagulability risk in bariatric patients contributed to HAT or if it was the innate risk of patients undergoing LT.

Studies in patients with LSG after LT have been promising. In a retrospective case-control study of patients undergoing LSG after LT, there was no significant difference in mean operative time or morbidity when compared with the non-LT LSG group. There were no conversions to open procedures, suggesting that prior LT did not increase complications as it relates to the surgical field. In patients with long-term follow-up, change in BMI after LSG was similar between LT and non-LT groups, although non-LT patients had significantly more excess body weight loss at 2 years. Importantly, LSG did not lead to changes in dosage of immunosuppressive mediations nor did it lead to liver complications. ${ }^{54}$

In the largest series to date, Morris et al demonstrated the safety, weight loss efficacy, and pronounced effect on the resolution of diabetes mellitus of LSG in post-LT recipients. ${ }^{55}$ Following LSG, there was a significant decrease in BMI (42.7 to $35.9 \mathrm{~kg} / \mathrm{m}^{2} ; p<0.01$ ), with up to a $20.6 \%$ total body weight loss at 1 -year follow-up. There was significant improvement in diabetes following LSG, as demonstrated by a significant decrease in the median daily insulin requirements (from 98 to 0 units/day; $p=0.02$ ), with $60 \%$ of patients able to discontinue insulin. A total of 15 patients underwent LSG following LT at a median time of 2.2 years between the two surgeries and a median follow-up period of 2.6 years. Most importantly, there was no liver allograft rejection after LSG and only one postoperative complication (surgical site infection). For patients undergoing LSG, post-LT patients had a similar decrease in BMI and reduction in comorbidities at 1 year compared with the matched non-LT patient cohort. This extended study experience highlights the relatively low morbidity and mortality in the delayed approach for LSG after transplant.

\section{Diabetes}

The relationship between NAFLD and T2DM is bidirectional; patients with NAFLD are at an increased risk of developing T2DM and those with T2DM are at an increased risk of developing NAFLD. ${ }^{56}$ In a cross-sectional study of 180 T2DM patients, the prevalence of steatosis found on ultrasound was $69.4 \%{ }^{57}$ NAFLD and T2DM are both strongly associated with features of MetS, which include insulin resistance, dyslipidemia, and obesity. There is growing evidence to support NAFLD and MetS sharing common genetic pathogenic mechanisms. ${ }^{58,59}$

Along with having higher prevalence of NALFD, patients with T2DM are at an increased risk of progression of NAFLD/NASH and development of fibrosis, cirrhosis, and HCC. $^{60,61}$ Therefore, it is not surprising that in patients with NASH who undergo LT, up to 70\% also carry a diagnosis of diabetes. ${ }^{62,63}$

Diabetes prior to LT is a strong predictor of early posttransplant complications, cardiovascular mortality, and overall decreased patient survival. ${ }^{64,65}$ In a retrospective cohort study of 12,442 patients who underwent LT, Hoehn et al reported longer hospital length of stay; higher peritransplant mortality, 30-day mortality, and 30-day readmission rates; and inferior graft and patient survival in those who had diabetes when compared with those without diabetes. ${ }^{66}$

There is currently no evidence to suggest that tightly controlled diabetes will improve survival rates. However preoperative hemoglobin A1c (HbA1C) has been shown to be predictive of posttransplant complications. In a small retrospective study with 173 patients, HbA1c $>7.3 \%$ was associated with a fourfold increased risk of biliary stricture formation. ${ }^{67}$

In pretransplant patients with diabetes, close monitoring and careful management of diabetes are crucial to minimize posttransplant complications and improve graft and patient survival.

\section{Renal Disease}

The burden of CKD still remains uncertain, with estimates of prevalence ranging widely from 4 to $42 \%{ }^{68}$ Despite the uncertainty, a continued increase in burden is expected, paralleling the rising prevalence of its risk factors including diabetes, obesity, MetS, hypertension, and aging. ${ }^{69}$ Patients with ESLD from cirrhosis and liver failure are at a risk of developing kidney injury. ${ }^{70}$ Patients who have ESLD from NAFLD are at even a higher risk of kidney injury. Given that NAFLD and CKD share many underlying risk factors, it is unsurprising that the two conditions are closely associated with one another. Experimental studies have suggested interlinked pathogenic mechanisms including serum proteins fetuin-A, adiponectin, and the energy sensor $5^{\prime}$-AMP-activated protein kinase (AMPK). ${ }^{71,72}$

There have been limited studies on NAFLD and CKD risk, but in the studies available, prevalence of CKD is increased in patients with NAFLD independent of age, sex, BMI, and other confounders such as diabetes, hypertension, and obesity. ${ }^{73-75}$ In a meta-analysis by Musso et al, patients with simple steatosis had increased incidence (hazard ratio: 1.79; 95\% confidence interval [CI]: 1.65-1.95) and prevalence (odds ratio: 2.12 ; 95\% CI: 1.69-2.66) of CKD risk. NAFLD disease progression was associated with even higher incidence and prevalence of CKD. NASH with advanced fibrosis 
was associated with higher incidence and prevalence of CKD than simple steatosis and nonadvanced fibrosis. ${ }^{76}$ A few years later, Mantovani et al also reported the association of NAFLD and CKD, finding nearly a $40 \%$ increase in the longterm risk of incident CKD in patients with NAFLD. ${ }^{77}$

CKD is prevalent in $46.8 \%$ of patients with cirrhosis admitted to the hospital. ${ }^{78}$ Patients with cirrhosis who have CKD have significantly higher rates of acute kidney injury (AKI), eventual need for dialysis, and reduced 30- and 90-day overall survival. ${ }^{78}$ Dependence on hemodialysis is a significant independent predictor of posttransplant survival. $^{79}$ Patients who are dialysis-dependent and are obese have a decreased 3-year posttransplant survival rate. ${ }^{63}$ In patients with both liver and kidney injuries, simultaneous liver/kidney (SLK) transplantation is often required. With the rising prevalence of NAFLD and CKD, NASH cirrhosis is the most rapidly growing indication for SLK transplantation. ${ }^{56}$ Unfortunately, patients with NASH have generally poor renal outcomes after SLK. ${ }^{2}$

\section{Cardiovascular Disease/Perioperative Risk}

CVD is the leading cause of nongraft-related mortality, accounting for up to $42 \%$ of nongraft-related deaths. ${ }^{80-83}$ LT imposes stresses on the cardiovascular system, such as increased blood pressure and peripheral vascular resistance after transplantation, which can unmask latent or clinically mild cirrhotic myocardial dysfunction, known as "cirrhotic cardiomyopathy." ${ }^{84}$ MetS is associated with an increased risk of CVD and accounts for up to one-third of CVD in men. ${ }^{85}$ The prevalence of MetS in patients after LT has been reported to be between 44 and 58\%, conferring an increased risk of cardiovascular complications. ${ }^{86,87}$

NAFLD is recognized as an independent risk factor of CVD. Vanwagner et al found that patients undergoing LT for NASH had higher rates of cardiovascular events within the first year after transplant compared with alcoholic cirrhosis patients even after controlling for comorbidities and a pretransplant history of cardiac disease. The majority of the events occurred in the perioperative period, but it was also found that cardiovascular complications do not appear to alter the overall survival of patients undergoing LT for cirrhosis resulting from NASH compared with alcohol. ${ }^{88}$

In a 2012 study, the cumulative risk of cardiovascular events post-LT was reported to be 4.5 and $10.1 \%$ at 1 and 3 years, respectively. The 1 - and 3-year risk was significantly higher in patients undergoing transplantation for NASH (15.3 and $19.3 \%$, respectively) as compared with all other etiologies. $^{89}$

Interestingly, coronary artery disease (CAD) does not seem to confer an increased risk of CVD mortality in transplant patients. $^{90}$ Patel et al recently reported that pre-LT CAD presence and severity did not impact survival after LT. This could be partly related to the fact that these patients were already being managed for CAD prior to LT and may have received more intensive monitoring after LT. The same group showed that diabetes was associated with an increased risk of having a cardiovascular event, and statin use demonstrated a survival benefit in patients who have undergone LT. ${ }^{90,91}$ This supports earlier studies demonstrating the association of MetS with CV complications, thus necessitating early identification and aggressive modification of MetS to improve outcomes.

Perioperative cardiac assessment of pretransplant patients with NASH is crucial to identify high-risk candidates who require medical optimization. There are currently no specific guidelines for the identification of CAD and CVD in pretransplant patients. ${ }^{92}$ The initial approach includes a clinical history to determine cardiovascular risk factors that include age, cigarette smoking, diabetes, dyslipidemia, and hypertension (- Table 1). ${ }^{93,94}$

All patients should undergo noninvasive pharmacological stress testing; dobutamine stress echocardiography is often preferred due to its high negative predictive value. In those who have equivocal or positive results on stress testing, coronary angiography is then recommended. Revascularization procedures should be considered in patients with significant stenosis, although there are no clear guidelines or recommendations in ESLD patients.

\section{Transplant Outcomes in NAFLD}

Patient and graft survival for patients who undergo LT for NASH cirrhosis are comparable with those of patients who undergo transplant for other etiologies. ${ }^{95}$ The 1-, 3- and 6-month survival for LT in NASH recipients are 94, 91, and $88 \%$, respectively. ${ }^{96}$ Posttransplant survival in patients with NASH at 1,3 , and 5 years was superior to the survival of patients with HCC, HCV, and alcohol-related liver disease, with rates reported at $87.6,82.2$, and $76.7 \%$, respectively. ${ }^{97}$

Post-LT NAFLD is a potential long-term complication that can occur as a recurrent disease in patients undergoing LT for

Table 1 Cardiovascular risk factor assessment

\begin{tabular}{|l|l|}
\hline Traditional cardiovascular risk factors & Coronary heart disease risk equivalents \\
\hline Age & Clinical coronary heart disease \\
\hline Sex & Symptomatic coronary artery disease \\
\hline Dyslipidemia & Peripheral artery disease \\
\hline Systolic blood pressure and the use of antihypertensive medications & Diabetes mellitus \\
\hline Current cigarette smoking & Chronic kidney disease \\
\hline Diabetes mellitus & Abdominal aortic aneurysm \\
\hline
\end{tabular}




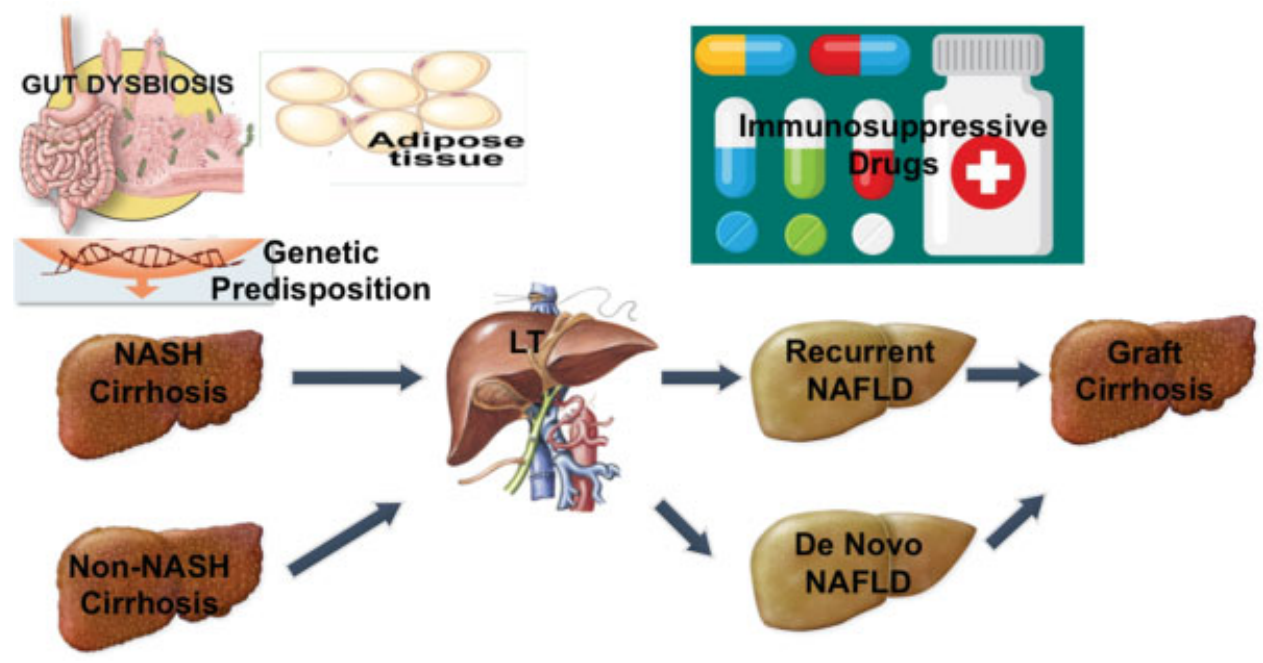

Fig. 1 NAFLD and liver transplantation (LT). NAFLD develops in genetically predisposed individuals due to multiple factors including obesity, insulin resistance, and gut dysbiosis. The disease may progress to cirrhosis requiring LT. NAFLD may recur after LT or may develop de novo in patients who were transplanted for other indications. Immunosuppressive drugs may contribute to the development of post-LT NAFLD. The natural history of post-LT NAFLD is not fully understood but the disease may eventually lead to graft cirrhosis. NAFLD, nonalcoholic fatty liver disease; NASH, nonalcoholic steatohepatitis.

NAFLD (recurrent post-LT NAFLD) or as a de novo complication in patients undergoing LT for indications other than NAFLD (de novo post-LT NAFLD) (- Fig. 1)..$^{98}$

\section{Recurrent Post-LT NAFLD}

A rising number of cases of recurrent NAFLD and NASH after LT have been reported. ${ }^{36}$ In a study of more than 200 transplant patients, Yalamanchili et al found that at 10 years, 33\% developed NAFLD. At 20 years, approximately $6 \%$ of patients developed NASH. ${ }^{99}$ Recurrence of NASH seems to demonstrate a distinctly different clinical evolution when compared with de novo NAFLD, exhibiting greater severity, more irreversibility, and an earlier onset. ${ }^{98}$ At 1-year post-LT, individuals transplanted for NASH cirrhosis have the highest risk of developing steatosis when compared with other etiologies including alcoholic cirrhosis, HCV, and cholestatic diseases (5\%). ${ }^{95,100}$ In a retrospective review of 588 adult LT recipients, Narayanan et al demonstrated that allograft steatosis at 10 years occurred in $77.6 \%$ of NASH recipients compared with $44.7 \%$ of non-

Table 2 Risk factors for recurrent NAFLD/NASH after liver transplantation

\begin{tabular}{|l|l|}
\hline Modifiable & Nonmodifiable \\
\hline Diabetes & Age \\
\hline Hypertension & $\begin{array}{l}\text { Genetics (e.g., genetic polymorphisms } \\
\text { PNPLA3) }\end{array}$ \\
\hline Hyperlipidemia & Sex \\
\hline $\begin{array}{l}\text { Increased BMI or } \\
\text { weight gain }\end{array}$ & Preexisting cardiovascular disease \\
\hline Renal dysfunction & \\
\hline
\end{tabular}

Abbreviations: BMI, body mass index; NAFLD, nonalcoholic fatty liver disease; NASH, nonalcoholic steatohepatitis; PNPLA3, patatin-like phospholipase domain-containing protein 3 .
NASH recipients, supporting the distinct difference between recurrent and de novo NAFLD post-LT. ${ }^{101}$

Factors that predict the presence of NAFLD in the pretransplant setting include older age, increased BMI and weight gain, and the presence of diabetes mellitus. ${ }^{102}$ Risk factors for the development of recurrent post-LT NAFLD can be categorized into modifiable versus nonmodifiable risk factors (-Table 2). ${ }^{103}$ Modifiable risk factors include diabetes, hypertension, hyperlipidemia, and renal dysfunction, whereas nonmodifiable risk factors include age, genetics, sex, and preexisting CVD.

Recent studies have shown that genetics play an important role in NAFLD, with special attention to patatin-like phospholipase domain-containing protein 3 (PNPLA3) polymorphism and interleukin $28 \mathrm{~B} .{ }^{104-106}$ In a recent study assessing the frequency of steatosis recurrence in the transplanted graft over a 5-year period, Finkenstedt et al demonstrated that the presence of PNPLA3 was associated with a greater prevalence of graft steatosis and increased risk of hepatic triglyceride accumulation. ${ }^{106}$

\section{De Novo Post-LT NAFLd}

Up to $56 \%$ of non-NASH transplant recipients develop posttransplant hepatic steatosis. ${ }^{107}$ In an early retrospective analysis by Seo et al, development of de novo NAFLD and de novo NASH occurred in 18 and 9\% of post-LT patients, respectively. ${ }^{108}$ Dumortier et al., reported a higher prevalence of post-LT NAFLD of $31 \%$, however the prevalence of steatohepatitis and severe fibrosis was low (5.3\% and $2.3 \%$, respectively). ${ }^{109}$ In a later study, Vallin et al reported the prevalence of steatohepatitis and severe fibrosis 5 years postLT as 12.5 and $17.2 \%$, respectively. ${ }^{98}$

Weight gain and obesity are significantly associated with de novo post-LT NAFLD. ${ }^{108,109}$ In later studies, additional risk factors were identified, which can be broadly characterized into recipient pre- and posttransplant risk factors. 
Table 3 Risk factors for de novo NAFLD/NASH after liver transplantation

\begin{tabular}{|l|l|}
\hline Pretransplant risk factors & Posttransplant risk factors \\
\hline Liver graft steatosis & Obesity \\
\hline $\begin{array}{l}\text { Alcoholic cirrhosis as } \\
\text { an indication for LT }\end{array}$ & Tacrolimus-based regimen \\
\hline & Diabetes mellitus \\
\hline & Hyperlipidemia \\
\hline & Hypertension \\
\hline
\end{tabular}

Abbreviations: LT, liver transplantation; NAFLD, nonalcoholic fatty liver disease; NASH, nonalcoholic steatohepatitis.

Posttransplant risk factors mirror the ones in recurrent postLT NAFLD: higher BMI, weight gain from time of transplant, the presence of diabetes, and higher HbA1c. Galvin et al conducted a retrospective single-center analysis in LT recipients, separately identifying pretransplant recipient risk factors to include older age, male sex, and HCV as indications for transplantation. ${ }^{110}$ Donor risk factors include older donor age and higher donor BMI (-Table 3). On multivariate analysis, BMI and tacrolimus were risk factors for the development of de novo post-LT NALFD. ${ }^{110}$

The use of donor livers with severe hepatic steatosis $(>60 \%)$ is associated with primary graft nonfunction. The use of donor livers with moderate-to-severe hepatic steatosis $(>30 \%)$ is associated with diminished outcomes including a significant decrease in 4-month graft survival (76 vs. $89 \%$; $p<0.05$ ) and in 2 -year patient survival (77 vs. 91\%; $p<0.05){ }^{111}$ Risk factors for donor liver steatosis include older age and the presence of MetS. Steatosis in donor livers may ultimately further exacerbate the donor shortage for LT. ${ }^{112}$

\section{Immunosuppression and Post-LT NAFLD}

Following transplant, the routine use of immunosuppressant drugs is known to enhance metabolic risk. Corticosteroids, calcineurin inhibitors, and mTOR (mammalian target of rapamycin) inhibitors all, to varying degrees, are involved in the development of insulin resistance (IR), diabetes, hypertension, obesity, and hyperlipidemia ( Table 4). ${ }^{113}$

Intuitively, the development of MetS could lead to NAFLD in the posttransplant setting.

Steroids are associated with hypertension, hyperglycemia, dyslipidemia, and obesity; thus, steroid-free regimens are favored. Increased levels of cholesterol and triglycerides are more frequently associated with cyclosporine. Posttransplant diabetes is more common with tacrolimus, probably due to more marked effects on the pancreatic $\beta$-cells. Sirolimus, a nonnephrotoxic drug, can also contribute independently to dyslipidemia. ${ }^{113}$ Thus, the general management rationale is to provide minimal immunosuppression to limit the possible side effects on the development of MetS, without risking graft rejection.

\section{Management of Recurrent/De Novo Post-LT NAFLD}

Currently, there are no established guidelines for the monitoring or treatment of NAFLD/NASH development or recurrence posttransplant. The prevention of recurrent NAFLD and NASH relies on the identification and management of risk factors such as obesity, diabetes, dyslipidemia, and hypertension. Aggressive risk factor modification in patients undergoing transplantation for NASH and in the pre- and posttransplant setting is crucial. Lifestyle modification, including prevention of weight gain and promotion of weight loss in those who are overweight or obese, is the first-line therapy for post-LT recurrent/de novo NAFLD. ${ }^{103}$

Assessment of posttransplant metabolic comorbidities and MetS should be routinely performed in transplant recipients as progression of graft fibrosis is significantly accelerated in immunosuppressed patients. ${ }^{114}$ General recommendations should include maintaining a healthy lifestyle and aggressive management of weight, diabetes, hypertension, dyslipidemia, and CVD (- Table 5).

No specific guidelines exist for follow-up for transplant recipients; thus, interval follow-up can be based on guidelines for the general population. However, clinicians must be cognizant of the increased risk of posttransplant metabolic complications so that early detection can ultimately lead to decreased incidences of recurrent and de novo NAFLD post-LT.

Evaluation of fibrosis and progression over time is important to ensure early identification of complications. The "gold standard" test, liver biopsy, has many limitations including cost, patient unwillingness, and risk of complications. This has led to the development of numerous noninvasive tools to detect and stage fibrosis. Serum markers (e.g., fibrosis-4 [FIB-4] and aspartate aminotransferase-toplatelet ratio index [APRI]) as well as imaging studies (e.g., TE and magnetic resonance elastography) have been validated as clinical tools in pretransplant NAFLD. ${ }^{115-119} \mathrm{TE}$ is a noninvasive method that is useful in detecting the degree of liver stiffness in patients with chronic liver disease. ${ }^{120}$ The

Table 4 Summary of immunosuppression and potential side effects

\begin{tabular}{|l|l|l|l|l|l|l|}
\hline & CS & Mycophenolate & Tacrolimus & Cyclosporine & Sirolimus & Everolimus \\
\hline Diabetes & a & b & c & d & d & d \\
\hline Hypertension & a & b & c & a & d & d \\
\hline Dyslipidemia & c & b & d & c & a & a \\
\hline Renal dysfunction & b & b & a & a & d & d \\
\hline
\end{tabular}

Abbreviation: CS, corticosteroids.

${ }^{\mathrm{a}}$ High risk, ${ }^{\mathrm{b}}$ No known association. ${ }^{\mathrm{C}}$ Moderate risk. ${ }^{\mathrm{d}}$ Least risk. 
Table 5 Recommendation and treatment aims in the posttransplant patient

\begin{tabular}{|l|}
\hline Maintain a healthy lifestyle \\
\hline Smoking cessation \\
\hline Routine physical activity \\
\hline Weight control \\
\hline Good control of blood pressure \\
\hline Tight control of glucose levels and diabetes \\
\hline Tight control of LDL cholesterol \\
\hline
\end{tabular}

Abbreviation: LDL, low-density lipoprotein.

accuracy of TE in diagnosing NAFLD and staging fibrosis in nontransplant patients has been extensively studied and validated. 121

There have been few published studies of these noninvasive tools in posttransplant recipients, and even fewer with posttransplant NAFLD patients. Many studies evaluating fibrosis in the post-LT setting have focused on HCVrelated disease and thus different proposed cutoff values when compared with NAFLD. Liver graft steatosis detection has been demonstrated with TE and controlled attenuation parameter (CAP), although the results were not validated against the "gold standard" of liver biopsy, and only $2 \%$ of the patients underwent transplant for the indication of NASH. ${ }^{122}$ In a meta-analysis by Bhat et al, the authors found that the APRI and FIB-4 can be used as estimates of significant fibrosis where TE is not available; however, TE is best to diagnose recurrent fibrosis in LT patients. ${ }^{123}$ However, the meta-analysis included studies where the etiology for transplant was primarily HCV. In a recent study, Galvin et al evaluated the diagnostic utility of noninvasive fibrosis scores such as FIB-4 and found limited utility of these scores in posttransplant NAFLD fibrosis evaluation. ${ }^{110}$

Unfortunately, there are no studies to date that compare the accuracy of TE for the diagnosis of NAFLD and fibrosis assessment with that for liver biopsy. Additionally, it is important to underscore the limitations of TE and how it impacts clinical practice, including liver position in instances of hemidiaphragmatic elevation or geometric mismatch of graft and abdominal cavity, and the lack of established cutoff values for the prediction of fibrosis or cirrhosis in the post-LT setting. 124

Although there are no FDA-approved medications for NAFLD/ NASH, several agents are being developed with some advancing to phase 3 trials. Obeticholic acid (OCA), which is an agonist for the farnesoid $X$ receptor (FXR), a nuclear receptor that senses bile acids and regulates their synthesis, is the most advanced drug in development. OCA showed consistent efficacy on fibrosis regression in phase 2 and 3 trials ${ }^{125,126}$ in patients with NASH and significant fibrosis, paving the way for potential FDA approval by 2020 . In the phase 3 REGENERATE trial (NCT02548351), patients who received OCA $25 \mathrm{mg}$ daily had significant improvement in fibrosis by 1 stage at 18 months compared with those in the placebo arm (23.1 vs. $11.9 \% ; p=0.0002$ ). Other drugs with different mechanisms of action have advanced to phase 3 as well, including elafibranor, cenicriviroc, resmetirom, aramchol, and saroglitazar. Although none of these drugs are being currently tested in the post-LT settings, these trials are likely to be conducted once the drugs are approved by the FDA in the general NASH population.

\section{Summary}

The prevalence of NAFLD has risen in parallel with the increasing prevalence of major risk factors including obesity, hypertension, T2DM, and hypertension. Unfortunately, with no FDA-approved medical treatments, NASH is rapidly becoming the leading indication for LT. Patients with NASH generally have medical comorbidities that require special consideration in the pretransplant setting. Medical optimization of risk factors such as obesity, CKD, and MetS will ultimately allow for improved LT outcomes. The presence of certain pretransplant risk factors such as obesity and diabetes increase the risk of posttransplant complications including recurrent NAFLD. De novo NAFLD is increasingly becoming an issue as donor organs are affected by the epidemic of obesity, diabetes, and NAFLD. Management of NAFLD posttransplant is similar to the management pretransplant. Early detection and intervention can prevent significant progression of disease.

\section{Disclosure}

Naim Alkhouri is on the speaker bureau for Intercept and he served as a consultant for Intercept, Allergan, and Zydus.

\section{Conflict of Interest}

Naim Alkhouri Eugenia Tsai received research funding from Intercept, Allergan, Genfit, Madrigal, Zydus, and Galmed.

\section{References}

1 European Association for the Study of the Liver (EASL); European Association for the Study of Diabetes (EASD); European Association for the Study of Obesity (EASO). EASL-EASD-EASO Clinical Practice Guidelines for the management of non-alcoholic fatty liver disease. J Hepatol 2016;64(06):1388-1402

2 Singal AK, Hasanin M, Kaif M, Wiesner R, Kuo YF. Nonalcoholic steatohepatitis is the most rapidly growing indication for simultaneous liver kidney transplantation in the United States. Transplantation 2016;100(03):607-612

3 Banini B, Mota M, Bhnke M, et al. NASH surpasses HCV as top etiology for adults listed for liver transplant. Presented at the American College of Gastroenterology Annual Scientific Meeting; October 14, 2016; Las Vegas, NV

4 Cholankeril G, Wong RJ, Hu M, et al. Liver transplantation for nonalcoholic steatohepatitis in the US: temporal trends and outcomes. Dig Dis Sci 2017;62(10):2915-2922

5 Jayakumar S. Liver transplantation for non-alcoholic fatty liver disease-a review. http://amj.amegroups.com/article/view/ 4320. Accessed January 1, 2018

6 Perumpail BJ, Khan MA, Yoo ER, Cholankeril G, Kim D, Ahmed A. Clinical epidemiology and disease burden of nonalcoholic fatty liver disease. World J Gastroenterol 2017;23(47): 8263-8276 
7 Giorgio V, Prono F, Graziano F, Nobili V. Pediatric non alcoholic fatty liver disease: old and new concepts on development, progression, metabolic insight and potential treatment targets. BMC Pediatr 2013;13:40

8 Papandreou D, Andreou E. Role of diet on non-alcoholic fatty liver disease: an updated narrative review. World J Hepatol 2015; 7(03):575-582

9 Tilg H, Moschen AR. Evolution of inflammation in nonalcoholic fatty liver disease: the multiple parallel hits hypothesis. Hepatology 2010;52(05):1836-1846

10 Noureddin M, Sanyal AJ. Pathogenesis of NASH: the impact of multiple pathways. Curr Hepatol Rep 2018;17(04):350-360

11 Kim WR, Lake JR, Smith JM, et al. OPTN/SRTR 2016 Annual Data Report: liver. Am J Transplant 2018;18(Suppl 1):172-253

12 Singal AK, Guturu P, Hmoud B, Kuo YF, Salameh H, Wiesner RH. Evolving frequency and outcomes of liver transplantation based on etiology of liver disease. Transplantation 2013;95(05): 755-760

13 Grundy SM, Cleeman JI, Daniels SR, et al; American Heart Association; National Heart, Lung, and Blood Institute. Diagnosis and management of the metabolic syndrome: an American Heart Association/National Heart, Lung, and Blood Institute Scientific Statement. Circulation 2005;112(17):2735-2752

14 Vernon G, Baranova A, Younossi ZM. Systematic review: the epidemiology and natural history of non-alcoholic fatty liver disease and non-alcoholic steatohepatitis in adults. Aliment Pharmacol Ther 2011;34(03):274-285

15 Calzadilla Bertot L, Adams LA. The natural course of non-alcoholic fatty liver disease. Int J Mol Sci 2016;17(05):774

16 Younossi ZM, Koenig AB, Abdelatif D, Fazel Y, Henry L, Wymer M. Global epidemiology of nonalcoholic fatty liver disease-Metaanalytic assessment of prevalence, incidence, and outcomes. Hepatology 2016;64(01):73-84

17 Rinella ME. Nonalcoholic fatty liver disease: a systematic review. JAMA 2015;313(22):2263-2273

18 Estes C, Razavi H, Loomba R, Younossi Z, Sanyal AJ. Modeling the epidemic of nonalcoholic fatty liver disease demonstrates an exponential increase in burden of disease. Hepatology 2018;67 (01):123-133

19 Mccullough A. The epidemiology and risk factors of NASH. In: Farrell GC, George J, de la M. Hall P, McCullough AJ. Fatty Liver Disease: NASH and Related Disorders. Malden, MA: Blackwell; 2007:23-37

20 Baffy G, Brunt EM, Caldwell SH. Hepatocellular carcinoma in non-alcoholic fatty liver disease: an emerging menace. J Hepatol 2012;56(06):1384-1391

21 Younossi ZM, Otgonsuren M, Henry L, et al. Association of nonalcoholic fatty liver disease (NAFLD) with hepatocellular carcinoma (HCC) in the United States from 2004 to 2009. Hepatology 2015;62(06):1723-1730

22 Berkan-Kawińska A, Piekarska A. Hepatocellular carcinoma in non-alcohol fatty liver disease - changing trends and specific challenges. Curr Med Res Opin 2019;1(October):1-9

23 Said A, Ghufran A. Epidemic of non-alcoholic fatty liver disease and hepatocellular carcinoma. World J Clin Oncol 2017;8(06): 429-436

24 Wong RJ, Aguilar M, Cheung R, et al. Nonalcoholic steatohepatitis is the second leading etiology of liver disease among adults awaiting liver transplantation in the United States. Gastroenterology 2015;148(03):547-555

25 Noureddin M, Vipani A, Bresee C, et al. NASH leading cause of liver transplant in women: updated analysis of indications for liver transplant and ethnic and gender variances. Am J Gastroenterol 2018;113(11):1649-1659

26 Siddique O, Joseph-Talreja M, Yoo ER, et al. Rising rate of liver transplantation in the baby boomer generation with non-alcoholic steatohepatitis in the United States. J Clin Transl Hepatol 2017;5(03):193-196
27 Cholankeril G, Yoo ER, Perumpail RB, et al. Rising rates of hepatocellular carcinoma leading to liver transplantation in baby boomer generation with chronic hepatitis C, alcohol liver disease, and nonalcoholic steatohepatitis-related liver disease. Diseases 2017;5(04):20

28 O'Leary JG, Landaverde C, Jennings L, Goldstein RM, Davis GL. Patients with NASH and cryptogenic cirrhosis are less likely than those with hepatitis $C$ to receive liver transplants. Clin Gastroenterol Hepatol 2011;9(08):700-704.e1

29 Thuluvath PJ, Hanish S, Savva Y. Waiting list mortality and transplant rates for NASH cirrhosis when compared with cryptogenic, alcoholic, or AIH cirrhosis. Transplantation 2019;103 (01):113-121

30 Flegal KM, Carroll MD, Kit BK, Ogden CL. Prevalence of obesity and trends in the distribution of body mass index among US adults, 1999-2010. JAMA 2012;307(05):491-497

31 Marchesini G, Brizi M, Morselli-Labate AM, et al. Association of nonalcoholic fatty liver disease with insulin resistance. Am J Med 1999;107(05):450-455

32 Charlton MR, Burns JM, Pedersen RA, Watt KD, Heimbach JK, Dierkhising RA. Frequency and outcomes of liver transplantation for nonalcoholic steatohepatitis in the United States. Gastroenterology 2011;141(04):1249-1253

33 LaMattina JC, Foley DP, Fernandez LA, et al. Complications associated with liver transplantation in the obese recipient. Clin Transplant 2012;26(06):910-918

34 Nair S, Vanatta JM, Arteh J, Eason JD. Effects of obesity, diabetes, and prior abdominal surgery on resource utilization in liver transplantation: a single-center study. Liver Transpl 2009;15 (11):1519-1524

35 Conzen KD, Vachharajani N, Collins KM, et al. Morbid obesity in liver transplant recipients adversely affects longterm graft and patient survival in a single-institution analysis. HPB (Oxford) 2015;17(03):251-257

36 Dureja P, Mellinger J, Agni R, et al. NAFLD recurrence in liver transplant recipients. Transplantation 2011;91(06):684-689

37 Tai C-M, Huang C-K, Hwang J-C, et al. Improvement of nonalcoholic fatty liver disease after bariatric surgery in morbidly obese Chinese patients. Obes Surg 2012;22(07):1016-1021

38 Lassailly G, Caiazzo R, Buob D, et al. Bariatric surgery reduces features of nonalcoholic steatohepatitis in morbidly obese patients. Gastroenterology 2015;149(02):379-388, quiz e15-e16

39 Praveen Raj P, Gomes RM, Kumar S, et al. The effect of surgically induced weight loss on nonalcoholic fatty liver disease in morbidly obese Indians: "NASHOST" prospective observational trial. Surg Obes Relat Dis 2015;11(06):1315-1322

40 Kwak M, Mehaffey JH, Hawkins RB, Hsu A, Schirmer B, Hallowell PT. Bariatric surgery is associated with reduction in non-alcoholic steatohepatitis and hepatocellular carcinoma: a propensity matched analysis. Am J Surg 2019:S0002-9610(19)30853-0

41 Froylich D, Corcelles R, Daigle C, Boules M, Brethauer S, Schauer P. Effect of Roux-en-Y gastric bypass and sleeve gastrectomy on nonalcoholic fatty liver disease: a comparative study. Surg Obes Relat Dis 2016;12(01):127-131

42 von Schönfels W, Beckmann JH, Ahrens M, et al. Histologic improvement of NAFLD in patients with obesity after bariatric surgery based on standardized NAS (NAFLD activity score). Surg Obes Relat Dis 2018;14(10):1607-1616

43 Mosko JD, Nguyen GC. Increased perioperative mortality following bariatric surgery among patients with cirrhosis. Clin Gastroenterol Hepatol 2011;9(10):897-901

44 Tichansky DS, Madan AK. Laparoscopic Roux-en-Y gastric bypass is safe and feasible after orthotopic liver transplantation. Obes Surg 2005;15(10):1481-1486

45 Nickel F, Tapking C, Benner L, et al. Bariatric surgery as an efficient treatment for non-alcoholic fatty liver disease in a prospective study with 1-year follow-up: BariScan study. Obes Surg 2018;28(05):1342-1350 
46 Luger M, Kruschitz R, Kienbacher C, et al. Prevalence of liver fibrosis and its association with non-invasive fibrosis and metabolic markers in morbidly obese patients with vitamin D deficiency. Obes Surg 2016;26(10):2425-2432

47 Lager CJ, Esfandiari NH, Subauste AR, et al. Roux-En-Y gastric bypass vs. sleeve gastrectomy: balancing the risks of surgery with the benefits of weight loss. Obes Surg 2017;27(01):154-161

48 Diwan TS, Lichvar AB, Leino AD, et al. Pharmacokinetic and pharmacogenetic analysis of immunosuppressive agents after laparoscopic sleeve gastrectomy. Clin Transplant 2017;31(06):

49 Heimbach JK, Watt KDS, Poterucha JJ, et al. Combined liver transplantation and gastric sleeve resection for patients with medically complicated obesity and end-stage liver disease. Am J Transplant 2013;13(02):363-368

50 Zamora-Valdes D, Watt KD, Kellogg TA, et al. Long-term outcomes of patients undergoing simultaneous liver transplantation and sleeve gastrectomy. Hepatology 2018;68(02):485-495

51 Klovaite J, Benn M, Nordestgaard BG. Obesity as a causal risk factor for deep venous thrombosis: a Mendelian randomization study. J Intern Med 2015;277(05):573-584

52 Pareja E, Cortes M, Navarro R, Sanjuan F, López R, Mir J. Vascular complications after orthotopic liver transplantation: hepatic artery thrombosis. Transplant Proc 2010;42(08):2970-2972

53 Duffy JP, Hong JC, Farmer DG, et al. Vascular complications of orthotopic liver transplantation: experience in more than 4,200 patients. J Am Coll Surg 2009;208(05):896-903, discussion 903-905

54 Tsamalaidze L, Stauffer JA, Arasi LC, et al. Laparoscopic sleeve gastrectomy for morbid obesity in patients after orthotopic liver transplant: a matched case-control study. Obes Surg 2018;28 (02):444-450

55 Morris MC, Jung AD, Kim Y, et al. Delayed sleeve gastrectomy following liver transplantation: a 5-year experience. Liver Transpl 2019;25(11):1673-1681

56 Kasturiratne A, Weerasinghe S, Dassanayake AS, et al. Influence of non-alcoholic fatty liver disease on the development of diabetes mellitus. J Gastroenterol Hepatol 2013;28(01):142-147

57 Leite NC, Salles GF, Araujo ALE, Villela-Nogueira CA, Cardoso CRL. Prevalence and associated factors of non-alcoholic fatty liver disease in patients with type-2 diabetes mellitus. Liver Int 2009; 29(01):113-119

58 Brunt EM, Wong VW-S, Nobili V, et al. Nonalcoholic fatty liver disease. Nat Rev Dis Primers 2015;1:15080

59 Cui J, Chen C-H, Lo M-T, et al; For The Genetics Of Nafld In Twins Consortium. Shared genetic effects between hepatic steatosis and fibrosis: a prospective twin study. Hepatology 2016;64(05): $1547-1558$

60 Loomba R, Abraham M, Unalp A, et al; Nonalcoholic Steatohepatitis Clinical Research Network. Association between diabetes, family history of diabetes, and risk of nonalcoholic steatohepatitis and fibrosis. Hepatology 2012;56(03):943-951

61 Zoppini G, Fedeli U, Gennaro N, Saugo M, Targher G, Bonora E. Mortality from chronic liver diseases in diabetes. Am J Gastroenterol 2014;109(07):1020-1025

62 Park CW, Tsai NT, Wong LL. Implications of worse renal dysfunction and medical comorbidities in patients with NASH undergoing liver transplant evaluation: impact on MELD and more. Clin Transplant 2011;25(06):E606-E611

63 Carter D, Dieterich DT, Chang C. Nonalcoholic fatty liver disease/nonalcoholic steatohepatitis in liver transplantation. Clin Liver Dis 2018;22(01):213-227

64 Yoo HY, Thuluvath PJ. The effect of insulin-dependent diabetes mellitus on outcome of liver transplantation. Transplantation 2002;74(07):1007-1012

65 Younossi ZM, Stepanova M, Saab S, et al. The impact of type 2 diabetes and obesity on the long-term outcomes of more than 85 000 liver transplant recipients in the US. Aliment Pharmacol Ther 2014;40(06):686-694
66 Hoehn RS, Singhal A, Wima K, et al. Effect of pretransplant diabetes on short-term outcomes after liver transplantation: a national cohort study. Liver Int 2015;35(07):1902-1909

67 Gray M, Singh S, Zucker SD. Influence of type 2 diabetes mellitus and preoperative hemoglobin A1c levels on outcomes of liver transplantation. Hepatol Commun 2019;3(04):574-586

68 McCullough K, Sharma P, Ali T, et al. Measuring the population burden of chronic kidney disease: a systematic literature review of the estimated prevalence of impaired kidney function. Nephrol Dial Transplant 2012;27(05):1812-1821

69 Stevens PE, Levin A; Kidney Disease: Improving Global Outcomes Chronic Kidney Disease Guideline Development Work Group Members. Evaluation and management of chronic kidney disease: synopsis of the kidney disease: improving global outcomes 2012 clinical practice guideline. Ann Intern Med 2013;158(11): 825-830

70 Singal AK, Ong S, Satapathy SK, Kamath PS, Wiesner RH. Simultaneous liver kidney transplantation. Transpl Int 2019;32(04): 343-352

71 Stefan N, Hennige AM, Staiger H, et al. Alpha2-Heremans-Schmid glycoprotein/fetuin-A is associated with insulin resistance and fat accumulation in the liver in humans. Diabetes Care 2006;29 (04):853-857

72 Ix JH, Sharma K. Mechanisms linking obesity, chronic kidney disease, and fatty liver disease: the roles of fetuin-A, adiponectin, and AMPK. J Am Soc Nephrol 2010;21(03):406-412

73 Targher G, Chonchol M, Bertolini L, et al. Increased risk of CKD among type 2 diabetics with nonalcoholic fatty liver disease. J Am Soc Nephrol 2008;19(08):1564-1570

74 Targher G, Bertolini L, Rodella S, Lippi G, Zoppini G, Chonchol M. Relationship between kidney function and liver histology in subjects with nonalcoholic steatohepatitis. Clin J Am Soc Nephrol 2010;5(12):2166-2171

75 Yasui K, Sumida Y, Mori Y, et al. Nonalcoholic steatohepatitis and increased risk of chronic kidney disease. Metabolism 2011;60 (05):735-739

76 Musso G, Gambino R, Tabibian JH, et al. Association of nonalcoholic fatty liver disease with chronic kidney disease: a systematic review and meta-analysis. PLoS Med 2014;11(07):e1001680

77 Mantovani A, Zaza G, Byrne CD, et al. Nonalcoholic fatty liver disease increases risk of incident chronic kidney disease: a systematic review and meta-analysis. Metabolism 2018;79:64-76

78 Wong F, Reddy KR, O'Leary JG, et al. Impact of chronic kidney disease on outcomes in cirrhosis. Liver Transpl 2019;25(06):870-880

79 Agopian VG, Kaldas FM, Hong JC, et al. Liver transplantation for nonalcoholic steatohepatitis: the new epidemic. Ann Surg 2012; 256(04):624-633

80 Therapondos G, Flapan AD, Plevris JN, Hayes PC. Cardiac morbidity and mortality related to orthotopic liver transplantation. Liver Transpl 2004;10(12):1441-1453

81 Ekstedt M, Franzén LE, Mathiesen UL, et al. Long-term follow-up of patients with NAFLD and elevated liver enzymes. Hepatology 2006;44(04):865-873

82 Pruthi J, Medkiff KA, Esrason KT, et al. Analysis of causes of death in liver transplant recipients who survived more than 3 years. Liver Transpl 2001;7(09):811-815

83 Vogt DP, Henderson JM, Carey WD, Barnes D. The long-term survival and causes of death in patients who survive at least 1 year after liver transplantation. Surgery 2002;132(04):775-780 , discussion 780

84 Myers RP, Lee SS. Cirrhotic cardiomyopathy and liver transplantation. Liver Transpl 2000;6(04, Suppl 1):S44-S52

85 Wilson PWF, D’Agostino RB, Parise H, Sullivan L, Meigs JB. Metabolic syndrome as a precursor of cardiovascular disease and type 2 diabetes mellitus. Circulation 2005;112(20):3066-3072

86 Bianchi G, Marchesini G, Marzocchi R, Pinna AD, Zoli M. Metabolic syndrome in liver transplantation: relation to etiology and immunosuppression. Liver Transpl 2008;14(11):1648-1654 
87 Hanouneh IA, Feldstein AE, McCullough AJ, et al. The significance of metabolic syndrome in the setting of recurrent hepatitis C after liver transplantation. Liver Transpl 2008;14(09): 1287-1293

88 Vanwagner LB, Bhave M, Te HS, Feinglass J, Alvarez L, Rinella ME. Patients transplanted for nonalcoholic steatohepatitis are at increased risk for postoperative cardiovascular events. Hepatology 2012;56(05):1741-1750

89 Albeldawi M, Aggarwal A, Madhwal S, et al. Cumulative risk of cardiovascular events after orthotopic liver transplantation. Liver Transpl 2012;18(03):370-375

90 Patel SS, Lin FP, Rodriguez VA, et al. The relationship between coronary artery disease and cardiovascular events early after liver transplantation. Liver Int 2019;39(07):1363-1371

91 Patel SS, Rodriguez VA, Siddiqui MB, et al. The impact of coronary artery disease and statins on survival after liver transplantation. Liver Transpl 2019;25(10):1514-1523

92 Ehtisham J, Altieri M, Salamé E, Saloux E, Ollivier I, Hamon M. Coronary artery disease in orthotopic liver transplantation: pretransplant assessment and management. Liver Transpl 2010;16(05):550-557

93 D'Agostino RBS Sr, Vasan RS, Pencina MJ, et al. General cardiovascular risk profile for use in primary care: the Framingham Heart Study. Circulation 2008;117(06):743-753

94 Sarnak MJ, Levey AS, Schoolwerth AC, et al; American Heart Association Councils on Kidney in Cardiovascular Disease, High Blood Pressure Research, Clinical Cardiology, and Epidemiology and Prevention. Kidney disease as a risk factor for development of cardiovascular disease: a statement from the American Heart Association Councils on Kidney in Cardiovascular Disease, High Blood Pressure Research, Clinical Cardiology, and Epidemiology and Prevention. Circulation 2003;108(17): 2154-2169

95 Maor-Kendler Y, Batts KP, Burgart LJ, et al. Comparative allograft histology after liver transplantation for cryptogenic cirrhosis, alcohol, hepatitis $\mathrm{C}$, and cholestatic liver diseases. Transplantation 2000;70(02):292-297

96 Pais R, Barritt AS IV, Calmus Y, et al. NAFLD and liver transplantation: current burden and expected challenges. J Hepatol 2016; 65(06):1245-1257

97 Afzali A, Berry K, Ioannou GN. Excellent posttransplant survival for patients with nonalcoholic steatohepatitis in the United States. Liver Transpl 2012;18(01):29-37

98 Vallin M, Guillaud O, Boillot O, Hervieu V, Scoazec JY, Dumortier J. Recurrent or de novo nonalcoholic fatty liver disease after liver transplantation: natural history based on liver biopsy analysis. Liver Transpl 2014;20(09):1064-1071

99 Yalamanchili K, Saadeh S, Klintmalm GB, Jennings LW, Davis GL. Nonalcoholic fatty liver disease after liver transplantation for cryptogenic cirrhosis or nonalcoholic fatty liver disease. Liver Transpl 2010;16(04):431-439

100 Molloy RM, Komorowski R, Varma RR. Recurrent nonalcoholic steatohepatitis and cirrhosis after liver transplantation. Liver Transpl Surg 1997;3(02):177-178

101 Narayanan P, Mara K, Izzy M, et al. Recurrent or de novo allograft steatosis and long-term outcomes after liver transplantation. Transplantation 2019;103(01):e14-e21

102 Angulo P, Keach JC, Batts KP, Lindor KD. Independent predictors of liver fibrosis in patients with nonalcoholic steatohepatitis. Hepatology 1999;30(06):1356-1362

103 Germani G, Laryea M, Rubbia-Brandt L, et al. Management of recurrent and de novo NAFLD/NASH after liver transplantation. Transplantation 2019;103(01):57-67

104 Watt KD, Dierkhising R, Fan C, et al. Investigation of PNPLA3 and IL28B genotypes on diabetes and obesity after liver transplantation: insight into mechanisms of disease. Am J Transplant 2013; 13(09):2450-2457
105 Petta S, Grimaudo S, Cammà C, et al. IL28B and PNPLA3 polymorphisms affect histological liver damage in patients with non-alcoholic fatty liver disease. J Hepatol 2012;56(06): 1356-1362

106 Finkenstedt A, Auer C, Glodny B, et al. Patatin-like phospholipase domain-containing protein 3 rs738409-G in recipients of liver transplants is a risk factor for graft steatosis. Clin Gastroenterol Hepatol 2013;11(12):1667-1672

107 Hejlova I, Honsova E, Sticova E, et al. Prevalence and risk factors of steatosis after liver transplantation and patient outcomes. Liver Transpl 2016;22(05):644-655

108 Seo S, Maganti K, Khehra M, et al. De novo nonalcoholic fatty liver disease after liver transplantation. Liver Transpl 2007;13(06): 844-847

109 Dumortier J, Giostra E, Belbouab S, et al. Non-alcoholic fatty liver disease in liver transplant recipients: another story of "seed and soil". Am J Gastroenterol 2010;105(03):613-620

110 Galvin Z, Rajakumar R, Chen E, et al. Predictors of de novo nonalcoholic fatty liver disease after liver transplantation and associated fibrosis. Liver Transpl 2019;25(01):56-67

111 Marsman WA, Wiesner RH, Rodriguez L, et al. Use of fatty donor liver is associated with diminished early patient and graft survival. Transplantation 1996;62(09):1246-1251

112 Orman ES, Barritt AS IV, Wheeler SB, Hayashi PH. Declining liver utilization for transplantation in the United States and the impact of donation after cardiac death. Liver Transpl 2013;19 (01):59-68

113 Barnard A, Konyn P, Saab S. Medical management of metabolic complications of liver transplant recipients. Gastroenterol Hepatol (N Y) 2016;12(10):601-608

114 Berenguer M. Host and donor risk factors before and after liver transplantation that impact HCV recurrence. Liver Transpl 2003; 9(11):S44-S47

115 Harrison SA, Oliver D, Arnold HL, Gogia S, Neuschwander-Tetri BA. Development and validation of a simple NAFLD clinical scoring system for identifying patients without advanced disease. Gut 2008;57(10):1441-1447

116 McPherson S, Stewart SF, Henderson E, Burt AD, Day CP. Simple non-invasive fibrosis scoring systems can reliably exclude advanced fibrosis in patients with non-alcoholic fatty liver disease. Gut 2010;59(09):1265-1269

117 Hashemi S-A, Alavian S-M, Gholami-Fesharaki M. Assessment of transient elastography (FibroScan) for diagnosis of fibrosis in non-alcoholic fatty liver disease: a systematic review and metaanalysis. Caspian J Intern Med 2016;7(04):242-252

118 Boursier J, Vergniol J, Guillet A, et al. Diagnostic accuracy and prognostic significance of blood fibrosis tests and liver stiffness measurement by FibroScan in non-alcoholic fatty liver disease. J Hepatol 2016;65(03):570-578

119 Besutti G, Valenti L, Ligabue G, et al. Accuracy of imaging methods for steatohepatitis diagnosis in non-alcoholic fatty liver disease patients: a systematic review. Liver Int 2019;39(08): 1521-1534

120 Fraquelli M, Rigamonti C, Casazza G, et al. Reproducibility of transient elastography in the evaluation of liver fibrosis in patients with chronic liver disease. Gut 2007;56(07):968-973

121 Tsai E, Lee T-P. Diagnosis and evaluation of nonalcoholic fatty liver disease/nonalcoholic steatohepatitis, including noninvasive biomarkers and transient elastography. Clin Liver Dis 2018;22(01):73-92

122 Karlas T, Kollmeier J, Böhm S, et al. Noninvasive characterization of graft steatosis after liver transplantation. Scand J Gastroenterol 2015;50(02):224-232

123 Bhat M, Tazari M, Sebastiani G. Performance of transient elastography and serum fibrosis biomarkers for non-invasive evaluation of recurrent fibrosis after liver transplantation: a metaanalysis. PLoS One 2017;12(09):e0185192 
30 Multidisciplinary Approach for Liver Transplantation in Patients with NASH Tsai et al.

124 Winters AC, Mittal R, Schiano TD. A review of the use of transient elastography in the assessment of fibrosis and steatosis in the postliver transplant patient. Clin Transplant 2019;33(10):e13700

125 Neuschwander-Tetri BA, Loomba R, Sanyal AJ, et al; NASH

Clinical Research Network. Farnesoid X nuclear receptor ligand obeticholic acid for non-cirrhotic, non-alcoholic steatohepatitis
(FLINT): a multicentre, randomised, placebo-controlled trial. Lancet 2015;385(9972):956-965

126 Younossi Z, Ratziu V, Loomba R, et al. GS-06-positive results from REGENERATE: a phase 3 international, randomized, placebocontrolled study evaluating obeticholic acid treatment for NASH. J Hepatol 2019;70(01):e5 